\title{
Magnetic Shift of the Chemical Freezeout and Electric Charge Fluctuations
}

\author{
Kenji Fukushima \\ Department of Physics, The University of Tokyo, \\ 7-3-1 Hongo, Bunkyo-ku, Tokyo 113-0033, Japan \\ Yoshimasa Hidaka \\ Theoretical Research Division, Nishina Center, RIKEN, Wako 351-0198, Japan
}

\begin{abstract}
We discuss the effect of a strong magnetic field on the chemical freezeout points in the ultrarelativistic heavy-ion collision. As a result of the inverse magnetic catalysis or the magnetic inhibition, the crossover onset to hot and dense matter out of quarks and gluons should be shifted to a lower temperature. To quantify this shift we employ the hadron resonance gas model and an empirical condition for the chemical freezeout. We point out that the charged particle abundances are significantly affected by the magnetic field so that the electric charge fluctuation is largely enhanced especially at high baryon density. The charge conservation partially cancels the enhancement but our calculation shows that the electric charge fluctuation and the charge chemical potential could serve as a magnetometer. We find that the fluctuation exhibits a crossover behavior rapidly increased for $e B \gtrsim(0.4 \mathrm{GeV})^{2}$, while the charge chemical potential has better sensitivity to the magnetic field.

PACS numbers: 25.75.-q, 25.75.Nq, 21.65.Qr, 12.38.-t
\end{abstract}

Introduction: Magnetic fields provide us with a useful probe to reveal non-trivial topological contents of the ground state of matter or the vacuum in quantum field theories. Dynamics of quarks, gluons, and composites is also significantly affected by an external magnetic field if its strength is comparable to the typical scale in quantum chromodynamics (QCD); i.e. $\Lambda_{\mathrm{QCD}} \sim 0.2 \mathrm{GeV}$. There are many works dedicated to strong magnetic field effects in the condensed matter and the neutron star environment $[1,2]$, in the early universe [3], and recently, more and more theoretical and experimental studies have been inspired by a possibility of gigantic magnetic fields created in the ultra-relativistic heavy-ion collision [4]. There are transport model simulations [5] that have verified an order estimate by simple classical modeling, leading to a compact formula [6, 7]: $e B(t)=e B_{0}\left[1+\left(t / t_{0}\right)^{2}\right]^{-3 / 2}$ with $e B_{0} \simeq(0.05 \mathrm{GeV})^{2}(1 \mathrm{fm} / b)^{2} Z \sinh Y$ where the atomic number is $Z=79$ in the Au-Au collision and the beam rapidity can be approximated as $\sinh Y \simeq \sqrt{s_{N N}} /\left(2 m_{N}\right) \simeq$ 107 at the top energy of Relativistic Heavy Ion Collider (RHIC) and $\sinh Y \simeq 2900$ at Large Hadron Collider (LHC). The impact parameter $b$ is of order of a few $\mathrm{fm}$ and so the largest magnetic field reaches as large as $e B_{0} \sim(\text { a few } \mathrm{GeV})^{2}$ (i.e. $B_{0} \sim 10^{20}$ gauss) or even larger, which must be the strongest magnetic field existing in the present universe. The life-time parameter $t_{0}$ decreases with increasing energy as $t_{0} \sim b /[2 \sinh Y]$ but it is important to note that the decay is not by exponential but by power and so at later time such as even $t \sim 10^{2} t_{0}$ the suppression factor is $\sim 10^{-6}$. Besides, there might be some additional mechanism to sustain the magnetic field from back-reaction of charged particles [8]. Also, it is worth mentioning that the quark matter ground state could be ferromagnetic [9], which would further enhance the initially generated magnetic field.
With such a hope of having a substantial residual portion out of the primordial magnetic field in the heavy-ion collision, lots of recent developments in the chiral kinetic theory [10] and the anomalous hydrodynamics [11] have been triggered. The coupling of almost massless (i.e. chiral) fermions and the external magnetic field is an intriguing setup for the investigation of exotic phenomena caused by quantum triangle anomalies. The chiral magnetic effect [12] (see Ref. [13] for a recent review) has attracted much interest from a broad range of physics and the impact has spread over condensed matter physics and astrophysics [14] (and historically speaking, the chiral magnetic effect was first recognized in the astrophysical context; see Ref. [15]). The fluctuation measurement of the electric charge separation is still ongoing at RHIC and LHC aimed at establishing the chiral magnetic effect in the heavy-ion collision, while the Weyl/Dirac semimetals can be cleaner devices for this purpose [16].

In parallel to such efforts to study topological effects, our understanding on bulk properties of QCD matter at strong $B$ has been advanced tremendously thanks to QCD-like theory calculations and the lattice-QCD simulations. It is an established fact that the chiral condensate increases with larger $B$ and this feature is commonly referred to as the magnetic catalysis [17]. One of the most striking and profound findings from the lattice-QCD simulation under large $B$ is what is called the inverse magnetic catalysis $[18,19]$ (which was originally used to refer to a response of high-density matter [20, 21]); with larger $B$ the chiral crossover takes place at smaller $T$ even though the chiral condensate at $T=0$ becomes larger. Usually the QCD vacuum is regarded as a BCS-like state and the critical temperature is naturally to be proportional to a $T=0$ condensate. Thus, all model-based calculations that favor a larger transition temperature 
at stronger $B$ have been challenged by this unexpected finding of the inverse magnetic catalysis.

There are some speculative scenarios to account for the finite- $T$ inverse magnetic catalysis. The interaction strength in chiral effective models may have some nontrivial dependence on $B$ so that this dependence, if the interaction gets weaker with larger $B$, can overcome the magnetic catalyzing effect (see Ref. [22] for a review and references therein). Such behavior of the interaction weakened by stronger $B$ may well be consistent with the asymptotic freedom of QCD if the relevant scale is given by $\sqrt{e B}[23,24]$. In other words, the inverse magnetic catalysis might be a consequence from the confining sector in which $B$ eases QCD particles of confining forces. An alternative scenario called the magnetic inhibition is rather closed in the chiral sector. If the magnetic field is large enough to resolve the internal fermionic contents of neutral mesons, the energy dispersion of $\pi^{0}$ is also dimensionally reduced, which would destruct the chiral order especially at finite $T$ [25]. There are also bag-model analyses of thermodynamic phase transitions with $B$ [26].

Inverse Magnetic Catalysis with the Hadron Resonance Gas Model: None of these model scenarios has been fully justified nor falsified and all of them suffer modeldependent assumptions. Fortunately, however, we have another theoretical tool, that is, the hadron resonance gas (HRG) model, which is free from parameter ambiguities. At zero magnetic field $(B=0)$ and zero baryon chemical potential $\left(\mu_{\mathrm{B}}=0\right)$ it has been well tested that the HRG model reproduces the lattice-QCD data very nicely up to the crossover temperature where the HRG thermodynamic quantities such as the pressure, the internal energy density, and the entropy density blow up. Interestingly, such a simple picture of the HRG model has been verified also from the success of thermal model fit of experimental data in the heavy-ion collision. In this way the chemical freezeout points have been located on the phase diagram on the $\mu_{\mathrm{B}}-T$ plane (see Ref. [27] for a summary of thermal model implications and Ref. [28] for recent studies on fluctuations to locate the chemical freezeout points).

It has been known that several thermodynamic conditions imposed with the HRG model can reproduce an experimentally identified curve of the chemical freezeout $[29,30]$. Among them a physically reasonable condition is $E / N=\varepsilon / n \simeq 1 \mathrm{GeV}$ where $E$ (and $\varepsilon$ ) is the internal energy (density) and $N$ (and $n$ ) is the thermal particle number (density) [29]. Here, $N$ counts not only baryons but also mesons and anti-particles. Therefore, the chemical freezeout supposedly occurs when the average energy per one thermal degrees of freedom (i.e. the rest mass plus thermally distributed energy $\sim m+\frac{3}{2} T$ for non-relativistic heavy particles) crosses $\sim 1 \mathrm{GeV}$. In Fig. 1 we show bands (with slanting lines) of the chemical freezeout using the HRG model in the range

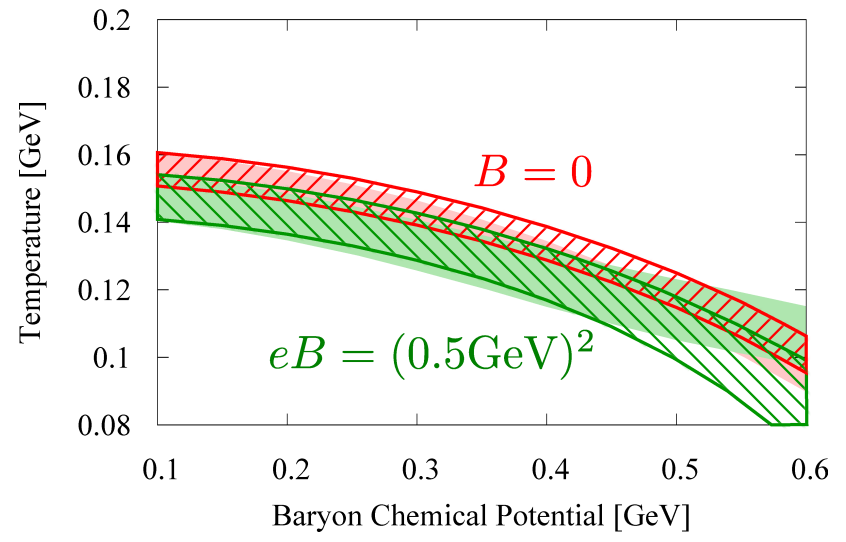

FIG. 1. Chemical freezeout bands drawn in the range of $E / N=0.9 \sim 1.0 \mathrm{GeV}$ with and without the magnetic field. The bands with slanting lines represent results with the charge conservation taken into account, while the shaded bands represent results with $\mu_{\mathrm{Q}}=\mu_{\mathrm{S}}=0$ fixed.

\begin{tabular}{|c|c|c|c|}
\hline & $a[\mathrm{GeV}]$ & $b\left[\mathrm{GeV}^{-1}\right]$ & $c\left[\mathrm{GeV}^{-3}\right]$ \\
\hline$B=0, E / N=0.9 \mathrm{GeV}$ & 0.1519 & 0.1347 & 0.05976 \\
$B=0, E / N=1.0 \mathrm{GeV}$ & 0.1618 & 0.1367 & 0.04705 \\
$e B=(0.5 \mathrm{GeV})^{2}, E / N=0.9 \mathrm{GeV}$ & 0.1418 & 0.1253 & 0.1849 \\
$e B=(0.5 \mathrm{GeV})^{2}, E / N=1.0 \mathrm{GeV}$ & 0.1555 & 0.1362 & 0.0565 \\
\hline
\end{tabular}

TABLE I. Chemical freezeout parameters for $E / N=0.9 \mathrm{GeV}$ and $1 \mathrm{GeV}$ with and without the magnetic field.

of $E / N=0.9 \sim 1.0 \mathrm{GeV}$ with and without the magnetic field. In our HRG model treatment we have adopted the particle data group list of particles contained in the package of THERMUS-V3.0 [31] (we used only the list and wrote our own numerical codes). We should note that we have introduced the strangeness and the electric charge chemical potentials, $\mu_{\mathrm{S}}$ and $\mu_{\mathrm{Q}}$, to implement the conservation laws of strangeness and electric charge for the entire system. More specifically, $\mu_{\mathrm{S}}$ and $\mu_{\mathrm{Q}}$ should take finite values to realize $N_{S}=0$ and $B /(2 Q)=1.2683$ where $B$ and $Q$ represent the baryon number and the electric charge number, respectively, which is for cold nuclear matter $\left(N_{\text {proton }}+N_{\text {neutron }}\right) / 2 N_{\text {proton }}$ and 1.2683 is fixed for heavy nuclei by the $\beta$-equilibrium with the Coulomb interaction.

The boundaries of the freezeout band (indicated by red lines for $B=0$ and green lines for $B \neq 0$ in Fig. 1) can be parametrized as a function of $\mu_{\mathrm{B}}$ in the polynomial form as $T_{\mathrm{f}}\left(\mu_{\mathrm{B}}\right)=a-b \mu_{\mathrm{B}}^{2}-c \mu_{\mathrm{B}}^{4}$. Then, we find that the choice of parameters as listed in Tab. I can give a good fit for the curves in Fig. 1. In fact, for $B=0$, these values determined from the $E / N$ condition with the HRG model are consistent with the results, $a=00.166 \pm 0.002 \mathrm{GeV}$, $b=0.139 \pm 0.016 \mathrm{GeV}^{-1}$, and $c=0.053 \pm 0.021 \mathrm{GeV}^{-3}$, fitted directly with the experimental data [30].

Hereafter we will take the strength of the magnetic field as $e B=(0.5 \mathrm{GeV})^{2}$ which may look a bit optimistic esti- 
mate but could possibly be sustained with back-reaction or even strengthened by ferromagnetism of high density matter. The condition of $E / N$ with $e B=(0.5 \mathrm{GeV})^{2}$ leads to a band shifted down to a lower temperature as shown by green lines in Fig. 1. This clearly means that the HRG model certainly encompasses the inverse magnetic catalysis as observed by the chiral condensate in the lattice-QCD simulation. We note that Ref. [32] already addressed how the HRG model can explain the (inverse) magnetic catalysis, but we should emphasize that it is non-trivial how the HRG model and the inverse magnetic catalysis would affect the freezeout curves. One would immediately understand this from the shaded bands in Fig. 1. The inverse magnetic catalysis implies that both $E$ and $N$ rapidly grow up at a lower temperature with stronger $B$, but it is not obvious which increases faster. Actually, if the baryon number density is large, the system is dominated by nucleons, so that $N$ (or the proton influenced by $B$ directly) increases faster. Thus, if the charge conservation is not imposed at high $\mu_{\mathrm{B}}$, the chemical freezeout curve as determined by a contour at constant $E / N$ should be pushed upward to a higher temperature by the $B$ effect, which is indicated by the shaded bands in Fig. 1. Below, we will discuss the effect of the charge conservation in more details.

Conservation Laws and Electric Charge Fluctuation: Now that we have identified the chemical freezeout curves on the phase diagram, we can estimate physical quantities along them predicting what should be seen in the experiment. Our central message here is that the fluctuation of electric charge is quite sensitive to the presence of $B \neq 0$, where the (dimensionless) electric charge fluctuation or susceptibility is defined as $\chi_{Q}=$ $T^{-2} \partial^{2} p / \partial \mu_{\mathrm{Q}}^{2}$ [33]. Figure 2 summarizes our results using the HRG model with and without $B$ and with and without the charge conservation. The red band with slanting lines represents $\chi_{Q}$ at $B=0$ and the band width corresponds to that in Fig. 1 with two freezeout conditions $E / N=0.9 \mathrm{GeV}$ and $1 \mathrm{GeV}$. The green band with slanting lines represents $\chi_{Q}$ at $e B=(0.5 \mathrm{GeV})^{2}$. A magnetic field increases $\chi_{Q}$ even at $\mu_{\mathrm{B}}=0$, which is testable in the lattice QCD simulation (see Ref. [34] for results consistent with our present calculation).

At larger $\mu_{\mathrm{B}}$ there are more neutrons and protons in the system. To keep the ratio, $B /(2 Q)=1.2683$, as fixed by the $\beta$-stability of heavy nuclei, we should introduce a negative isospin chemical potential or a negative charge chemical potential $\mu_{\mathrm{Q}}<0$. As long as the isospin symmetry approximately holds at $B=0$, the charge chemical potential $\mu_{\mathrm{Q}}$ remains of order of $\sim 0.01 \mathrm{GeV}$ even at $\mu_{\mathrm{B}} \sim 0.6 \mathrm{GeV}$ as seen from $\mu_{\mathrm{Q}}[B=0]$ in Fig. 3. Therefore, for $B=0$, it should be an acceptable approximation to neglect the effect of the electric charge conservation at all. However, the strangeness conservation must be properly implemented with $\mu_{\mathrm{S}}$, which is clearly under-

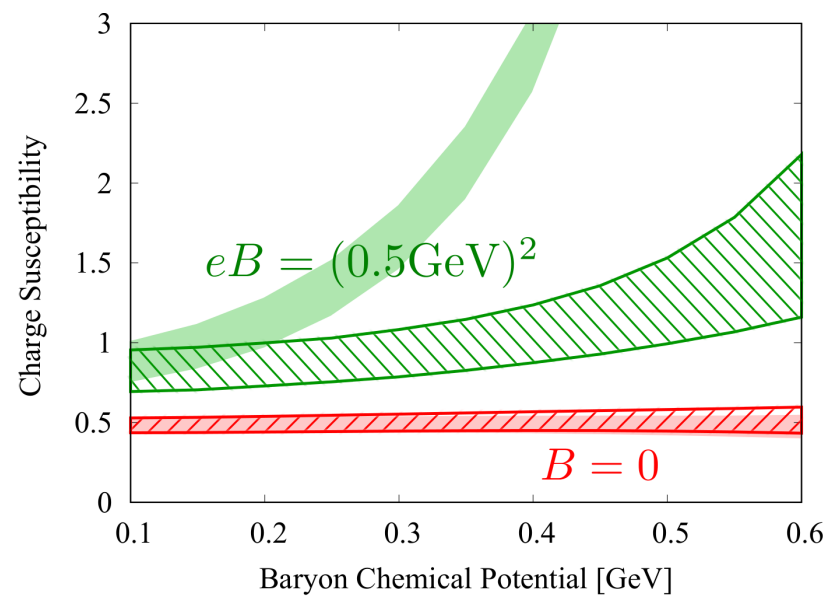

FIG. 2. Electric charge susceptibility along the chemical freezeout lines, on which the location is indicated as a function of $\mu_{\mathrm{B}}$, with and without $B$. The bands with slanting lines represent results with the charge conservation taken into account, while the shaded bands represent results with $\mu_{\mathrm{Q}}=\mu_{\mathrm{S}}=0$ fixed.

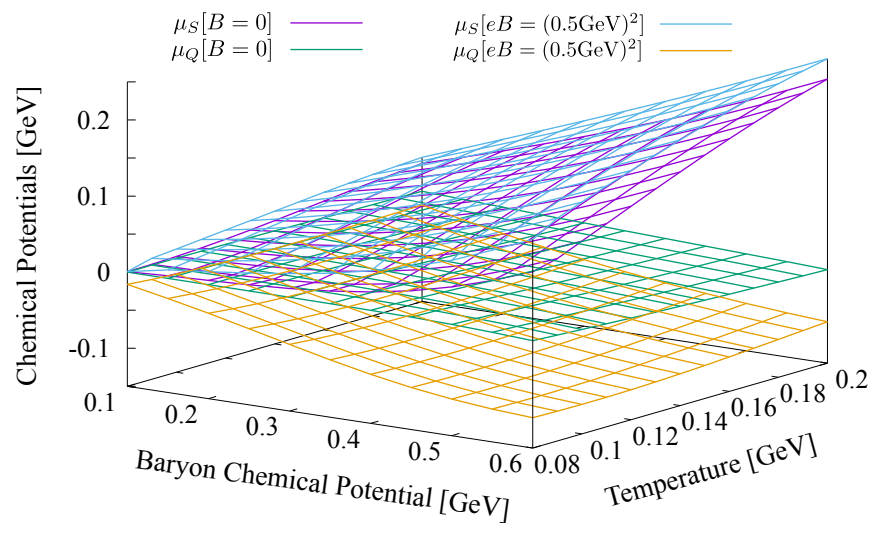

FIG. 3. Chemical potentials, $\mu_{\mathrm{S}}$ and $\mu_{\mathrm{Q}}$, determined from the HRG model and conservation laws, $n_{S}=0$ and $B /(2 Q)=$ 1.2683 , as functions of $\mu_{\mathrm{B}}$ and $T$ with and without $B$.

stood from $\mu_{\mathrm{S}}[B=0]$ in Fig. 3. Actually, for $B=0$ and around $T \sim 0.2 \mathrm{GeV}$, we have found $\mu_{\mathrm{S}} \sim \mu_{\mathrm{B}} / 3$ (= quark chemical potential) as expected in the deconfined phase. (We note that the realization of $\mu_{\mathrm{S}} \sim \mu_{\mathrm{B}} / 3$ is physically natural, but highly non-trivial in the HRG model because there is no quark degrees of freedom explicitly contained in the model.) Because strange quarks have $S=-1$, the total chemical potential felt by strange quarks becomes vanishing for $\mu_{\mathrm{S}}=\mu_{\mathrm{B}} / 3$, so that the strangeness density is zero then.

Let us consider what happens for a strong- $B$ situation. Naturally, the isospin symmetry is explicitly broken, and because the phase-space density is proportional to the Landau degeneracy factor $e B /(2 \pi)$ the proton density is more favored than the neutron density as $B$ increases. Then, there are more protons in the system, causing that the electric charge fluctuation is also enhanced at 


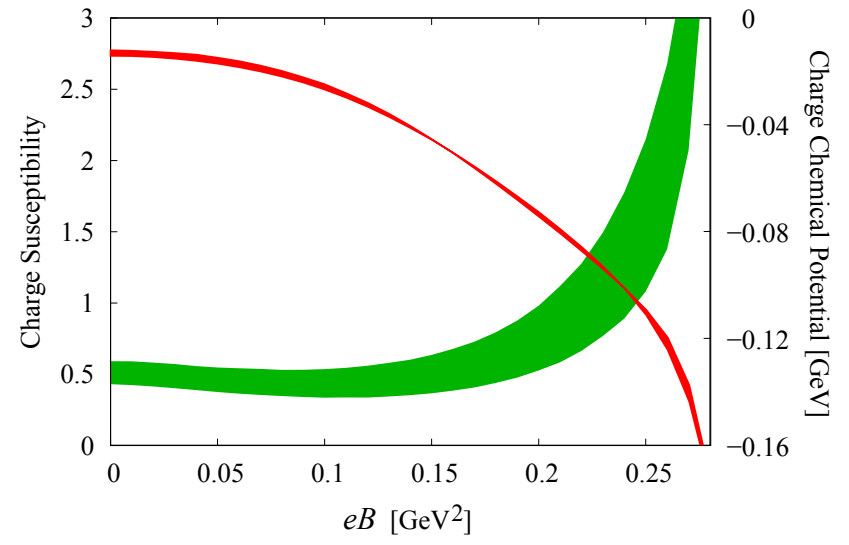

FIG. 4. $\chi_{Q}$ (green line increasing toward the top-right corner) and $\mu_{\mathrm{Q}}$ (red line decreasing toward the bottom-right corner) as functions of $e B$ for $\mu_{\mathrm{B}}=0.6 \mathrm{GeV}$. The band corresponds to $E / N=0.9 \mathrm{GeV}$ and $1.0 \mathrm{GeV}$ and the temperature is fixed by respective freezeout conditions.

stronger $B$, as is represented by the green shaded band in Fig. 2. As $\mu_{\mathrm{B}}$ goes up from $0.1 \mathrm{GeV}$ to $0.6 \mathrm{GeV}$, our calculation shows that $\chi_{Q}$ rapidly increases by more than one order of magnitude. However, such a gigantic enhancement is an artifact from too abundant protons over neutrons, which violates the electric charge conservation. Indeed, for a stronger $B$, as seen from $\mu_{\mathrm{Q}}\left[e B=(0.5 \mathrm{GeV})^{2}\right]$ in Fig. 3, a larger value of $\mu_{\mathrm{Q}}$ of order of $\sim 0.1 \mathrm{GeV}$ is necessary to suppress protons not to violate the charge conservation. This chemical potential also suppresses $\chi_{Q}$ at large $\mu_{\mathrm{B}}$. To have an intuitive feeling of how results change quantitatively, we show all the cases with and without $B$ and with and without the charge conservation in Fig. 2 corresponding to the presentation in Fig. 1. We see from Fig. 2 that the conservation laws have minor effects for $B=0$ (and we can also understand the strangeness sector hardly modifies $\left.\chi_{Q}\right)$, but the proper treatment with the conservation laws is indispensable for $e B=(0.5 \mathrm{GeV})^{2}$. Nevertheless, even after imposing the conservation laws, we can confirm that $\chi_{Q}$ at $e B=(0.5 \mathrm{GeV})^{2}$ is twice enhanced for small $\mu_{\mathrm{B}}$ and, for $\mu_{\mathrm{B}} \sim 0.6 \mathrm{GeV}$ it can be four times as large as that at $B=0$, that implies that $\chi_{Q}$ could serve as a probe to detect the presence of a strong magnetic field in the heavy-ion collision.

We make another plot in Fig. 4 to check the sensitivity of $\chi_{Q}$ and $\mu_{\mathrm{Q}}$ as functions of $e B$ for $\mu_{\mathrm{B}}=0.6 \mathrm{GeV}$. These are surprisingly non-trivial results; $\chi_{Q}$ is almost flat up to $e B \sim 0.15 \mathrm{GeV}^{2}$ and a quick "crossover" of increasing $\chi_{Q}$ takes place for $e B \gtrsim(0.4 \mathrm{GeV})^{2}$. The rapid increasing behavior in Fig. 4 suggests a sort of approximate phase transition and quark matter at $e B \gtrsim(0.4 \mathrm{GeV})^{2}$ might be well identified as a novel state of matter. It would be an interesting future problem to explore intrinsic properties of such dense and magnetized matter. From the experimental point of view, $\mu_{\mathrm{Q}}$ should be a probe that has better sensitivity to the magnetic field. Practically speaking, moreover, the determination of $\mu_{\mathrm{Q}}$ would need less statistics than the fluctuation measurements.

Summary: We quantified a shift of the chemical freezeout curves by the magnetic field effect using the HRG model and the freezeout condition $E / N \sim 1 \mathrm{GeV}$. We found that the chemical freezeout shift is downward to a lower temperature in a way consistent with the inverse magnetic catalysis, though physical contents may be different; we focused on a combination of $E / N$ and the electric charge chemical potential $\mu_{\mathrm{Q}}$ plays a crucial role at large $\mu_{\mathrm{B}}$ and strong $B$, not to violate the electric charge conservation. We proposed an enhancement of the electric charge fluctuation $\chi_{Q}$ as a probe to detect $B$ in the heavy-ion collision. Alternatively, the proton number fluctuation could be useful because such an enhancement is mainly caused by protons whose dispersion should be drastically modified by the magnetic field. Also, in principle, $\mu_{\mathrm{Q}}$ is an experimentally measurable quantity from the thermal model fit, and increasing $\mu_{\mathrm{Q}}$ could serve as a magnetometer. In particular, we found that there is rapidly increasing behavior of fluctuation around $e B \sim(0.4 \mathrm{GeV})^{2}$ which may signal for a crossover to a yet unknown new state of matter.

A possible experimental analysis would be as follows. There are already nice collections of the thermal model parameters, $T, \mu_{\mathrm{B}}, \mu_{\mathrm{Q}}, \mu_{\mathrm{S}}$, etc from the beam energy scan program at RHIC and more data should be expected from the future heavy-ion facilities such as FAIR, NICA, and JPARC. These fitted parameters should generally have centrality dependence, and once this dependence together with the electric charge fluctuation can be resolved experimentally, our present calculation suggests that we can extract information on the magnetic field. In other words, we emphasize the importance of centrality differentiated measurement of thermal parameters and fluctuations, and our present study provides future experiments with a theoretical baseline for the magnetic field detection. Finally, we would emphasize that, even apart from pragmatic discussions on experimental opportunities, our present study sheds a new light of theory on the phase structure and the ground state properties of highly magnetized QCD matter.

K. F. thanks Vladimir Skokov and Igor Shovkovy for discussions. This work was partially supported by JSPS KAKENHI Grant No. 15H03652 (K. F. and Y. H.), $15 \mathrm{~K} 13479$ (K. F.), and $16 \mathrm{~K} 17716$ (Y. H.) and partially by the RIKEN interdisciplinary Theoretical Science (iTHES) project.

[1] D. Lai, Rev. Mod. Phys. 73, 629 (2001), arXiv:astroph/0009333 [astro-ph]. 
[2] V. A. Miransky and I. A. Shovkovy, Phys. Rept. 576, 1 (2015), arXiv:1503.00732 [hep-ph].

[3] K. Subramanian, Astron. Nachr. 331, 110 (2010), arXiv:0911.4771 [astro-ph.CO].

[4] X.-G. Huang, (2015), arXiv:1509.04073 [nucl-th].

[5] V. Skokov, A. Illarionov, and V. Toneev, Int. J. Mod. Phys. A24, 5925 (2009), arXiv:0907.1396 [nucl-th]; W.T. Deng and X.-G. Huang, Phys. Rev. C85, 044907 (2012), arXiv:1201.5108 [nucl-th].

[6] D. E. Kharzeev and H. J. Warringa, Phys. Rev. D80, 034028 (2009), arXiv:0907.5007 [hep-ph].

[7] K. Fukushima, J. Phys. G39, 013101 (2012), arXiv:1108.2939 [hep-ph].

[8] K. Tuchin, Phys. Rev. C88, 024911 (2013), arXiv:1305.5806 [hep-ph].

[9] T. Tatsumi, Phys. Lett. B489, 280 (2000), arXiv:hepph/9910470 [hep-ph].

[10] M. A. Stephanov and Y. Yin, Phys. Rev. Lett. 109, 162001 (2012), arXiv:1207.0747 [hep-th].

[11] D. T. Son and P. Surowka, Phys. Rev. Lett. 103, 191601 (2009), arXiv:0906.5044 [hep-th].

[12] K. Fukushima, D. E. Kharzeev, and H. J. Warringa, Phys. Rev. D78, 074033 (2008), arXiv:0808.3382 [hep$\mathrm{ph}$.

[13] J. Liao, in 25th International Conference on UltraRelativistic Nucleus-Nucleus Collisions (Quark Matter 2015) Kobe, Japan, September 27-October 3, 2015 (2016) arXiv:1601.00381 [nucl-th].

[14] N. Yamamoto, Phys. Rev. D93, 065017 (2016), arXiv:1511.00933 [astro-ph.HE].

[15] A. Vilenkin, Phys. Rev. D22, 3080 (1980); M. A. Metlitski and A. R. Zhitnitsky, Phys. Rev. D72, 045011 (2005), arXiv:hep-ph/0505072 [hep-ph].

[16] Q. Li, D. E. Kharzeev, C. Zhang, Y. Huang, I. Pletikosic, A. V. Fedorov, R. D. Zhong, J. A. Schneeloch, G. D. Gu, and T. Valla, (2014), 10.1038/nphys3648, arXiv:1412.6543 [cond-mat.str-el].

[17] K. Klimenko, Theor. Math. Phys. 89, 1161 (1992); Theor. Math. Phys. 90, 1 (1992); V. Gusynin, V. Miransky, and I. Shovkovy, Phys. Rev. Lett. 73, 3499 (1994), arXiv:hep-ph/9405262 [hep-ph]; Phys. Rev. D52, 4718 (1995), arXiv:hep-th/9407168 [hep-th]; Phys. Lett. B349, 477 (1995), arXiv:hep-ph/9412257 [hep-ph]; Nucl. Phys. B462, 249 (1996), arXiv:hep-ph/9509320 [hep-ph]; I. Shushpanov and A. V. Smilga, Phys. Lett. B402, 351 (1997), arXiv:hep-ph/9703201 [hep-ph].

[18] G. Bali, F. Bruckmann, G. Endrodi, Z. Fodor, S. Katz, et al., JHEP 1202, 044 (2012), arXiv:1111.4956 [heplat]; G. S. Bali, F. Bruckmann, G. Endrodi, Z. Fodor, S. D. Katz, and A. Schafer, Phys. Rev. D86, 071502
(2012), arXiv:1206.4205 [hep-lat]; G. S. Bali, F. Bruckmann, G. Endrodi, S. D. Katz, and A. Schafer, JHEP 08, 177 (2014), arXiv:1406.0269 [hep-lat].

[19] L. Levkova and C. DeTar, Phys. Rev. Lett. 112, 012002 (2014), arXiv:1309.1142 [hep-lat]; V. G. Bornyakov, P. V. Buividovich, N. Cundy, O. A. Kochetkov, and A. Schafer, Phys. Rev. D90, 034501 (2014), arXiv:1312.5628 [hep-lat].

[20] F. Preis, A. Rebhan, and A. Schmitt, JHEP 1103, 033 (2011), arXiv:1012.4785 [hep-th]; (2012), arXiv:1208.0536 [hep-ph].

[21] K. Fukushima and J. M. Pawlowski, (2012), arXiv:1203.4330 [hep-ph].

[22] R. Gatto and M. Ruggieri, Lect. Notes Phys. 871, 87 (2013), arXiv:1207.3190 [hep-ph].

[23] J. Braun, W. A. Mian, and S. Rechenberger, Phys. Lett. B755, 265 (2016), arXiv:1412.6025 [hep-ph].

[24] N. Mueller and J. M. Pawlowski, Phys. Rev. D91, 116010 (2015), arXiv:1502.08011 [hep-ph].

[25] K. Fukushima and Y. Hidaka, Phys. Rev. Lett. 110, 031601 (2013), arXiv:1209.1319 [hep-ph].

[26] E. S. Fraga and L. F. Palhares, Phys. Rev. D86, 016008 (2012), arXiv:1201.5881 [hep-ph]; E. S. Fraga, J. Noronha, and L. F. Palhares, (2012), arXiv:1207.7094 [hep-ph].

[27] A. Andronic et al., Nucl. Phys. A837, 65 (2010), arXiv:0911.4806 [hep-ph].

[28] P. Alba, W. Alberico, R. Bellwied, M. Bluhm, V. Mantovani Sarti, M. Nahrgang, and C. Ratti, Phys. Lett. B738, 305 (2014), arXiv:1403.4903 [hep-ph]; P. Alba, R. Bellwied, M. Bluhm, V. Mantovani Sarti, M. Nahrgang, and C. Ratti, Phys. Rev. C92, 064910 (2015), arXiv:1504.03262 [hep-ph].

[29] J. Cleymans and K. Redlich, Phys. Rev. Lett. 81, 5284 (1998), arXiv:nucl-th/9808030 [nucl-th].

[30] J. Cleymans, H. Oeschler, K. Redlich, and S. Wheaton, Phys. Rev. C73, 034905 (2006), arXiv:hep-ph/0511094 [hep-ph].

[31] S. Wheaton and J. Cleymans, Comput. Phys. Commun. 180, 84 (2009), arXiv:hep-ph/0407174 [hep-ph].

[32] G. Endrodi, JHEP 04, 023 (2013), arXiv:1301.1307 [hep$\mathrm{ph}$.

[33] F. Karsch and K. Redlich, Phys. Lett. B695, 136 (2011), arXiv:1007.2581 [hep-ph]; B. Friman, F. Karsch, K. Redlich, and V. Skokov, Eur. Phys. J. C71, 1694 (2011), arXiv:1103.3511 [hep-ph].

[34] P. V. Buividovich, M. N. Chernodub, E. V. Luschevskaya, and M. I. Polikarpov, Phys. Rev. D80, 054503 (2009), arXiv:0907.0494 [hep-lat]. 